\title{
A Comparative Analysis of have a look at and take a look at in Four Corpora
}

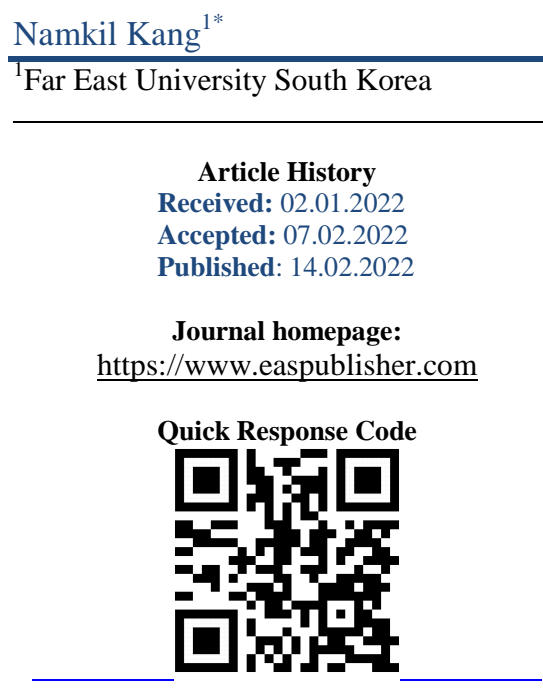

\begin{abstract}
The ultimate goal of this paper is to provide a comparative analysis of have a look at and take a look at in four corpora. A major point to note is that take a look at is preferred over have a look at in the TV Corpus from the 1950s to the 2010s. A further point to note is two types reached a peak in the 2010s, which in turn implies that they are the most preferred ones in the 2010s. With respect to the Movie Corpus, it is worth noting that take a look at is favored over have a look at in the Movie Corpus from the 1930s to the 2010s. It is noteworthy, on the other hand, have a look at reached a peak (346 tokens) in the 2000s, whereas take a look at reached a peak ( 1,310 tokens) in the 2010s. This in turn implies that two types were the most preferred ones in in those periods. The COCA clearly shows that have a look at http is the most preferred one in America, whereas take a look at it is the most preferred one. The COCA also shows that $8.88 \%$ of forty five nouns are the collocations of both have a look at and take a look at. The BNC clearly indicates that have a look at number and take a look at trends are the most preferable ones among the British. With respect to the BNC, it is interesting to point out that only $3.7 \%$ of twenty seven nouns are the collocation of have a look at and take a look at. Keywords: Corpus, type, token, COCA, BNC, TV Corpus, Movie Corpus, have a look at, take a look at.
\end{abstract}

Copyright (C) 2022 The Author(s): This is an open-access article distributed under the terms of the Creative Commons Attribution 4.0 International License (CC BY-NC 4.0) which permits unrestricted use, distribution, and reproduction in any medium for non-commercial use provided the original author and source are credited.

\section{INTRODUCTION}

As Murphy (2016, 2019) points out, have a look at and take a look at are used interchangeably. The main goal of this paper is to provide a comparative analysis of have a look at and take a look at in four corpora. We consider which type is the preferable one in the TV Corpus and the Movie Corpus. Also, we consider the collocations of have a look at and take a look at in the COCA and BNC. We examine which collocations are the preferable ones among Americans and the British. The organization of this paper is as follows. In section 2, we argue that take a look at is preferred over have a look at in the TV Corpus from the 1950 s to the 2010s. Two types reached a peak in the 2010s, which in turn implies that they are the most preferred ones in the 2010s. In section 3, we maintain that take a look at is favored over have a look at in the Movie Corpus from the 1930s to the 2010s. Interestingly, have a look at reached a peak (346 tokens) in the 2000s, whereas take a look at reached a peak (1,310 tokens) in the 2010s, which in turn implies that two types were the most preferred ones in in those periods. In section 4, we contend that have a look at http is the most preferred one in America, whereas take a look at it is the most preferred one. It is important to note that $8.88 \%$ of forty five nouns are the collocations of both have a look at and take a look at in the COCA. In section 5, we argue that have a look at number and take a look at trends are the most preferable ones among the British. Additionally, we show that only $3.7 \%$ of twenty seven nouns are the collocation of have a look at and take a look at in the BNC.

\section{The TV Corpus}

In what follows, we consider a comparative analysis of have a look at and take a look at in the TV Corpus. The TV Corpus includes the TV programs of America, the UK, Canada, Australia, New Zealand, and Ireland. In addition, it includes a database from the 1950 s to the 2010s. Table 1 shows the use and frequency of have a look at and take a look at in the TV programs of six countries from the 1950s to the 2010s: 
Table-1: Frequency of have a look at and take a look at in the TV Corpus

\begin{tabular}{|l|l|l|}
\hline Type & Have a look at & Take a look at \\
\hline $1950 \mathrm{~s}$ & 17 & 65 \\
\hline $1960 \mathrm{~s}$ & 176 & 310 \\
\hline $1970 \mathrm{~s}$ & 184 & 325 \\
\hline $1980 \mathrm{~s}$ & 187 & 468 \\
\hline $1990 \mathrm{~s}$ & 236 & 988 \\
\hline $2000 \mathrm{~s}$ & 598 & 2,688 \\
\hline $2010 \mathrm{~s}$ & 1,188 & 4,429 \\
\hline All & 2,586 & 9,273 \\
\hline US/CA & 904 & 8,117 \\
\hline UK/IE & 1,548 & 987 \\
\hline AU/NZ & 122 & 104 \\
\hline Misc & 12 & 65 \\
\hline
\end{tabular}

An important question is "Which type is the preferable one? As illustrated in Table 1, the overall frequency of have a look at is 2,586 tokens, whereas that of take a look at is 9,273 tokens. That is to say, the frequency of have a look at is three times higher than that of take a look at. This in turn suggests that take a look at is favored over have a look at. The following graph shows how much have a look at and take a look at are used from the 1950s to the 2010s:

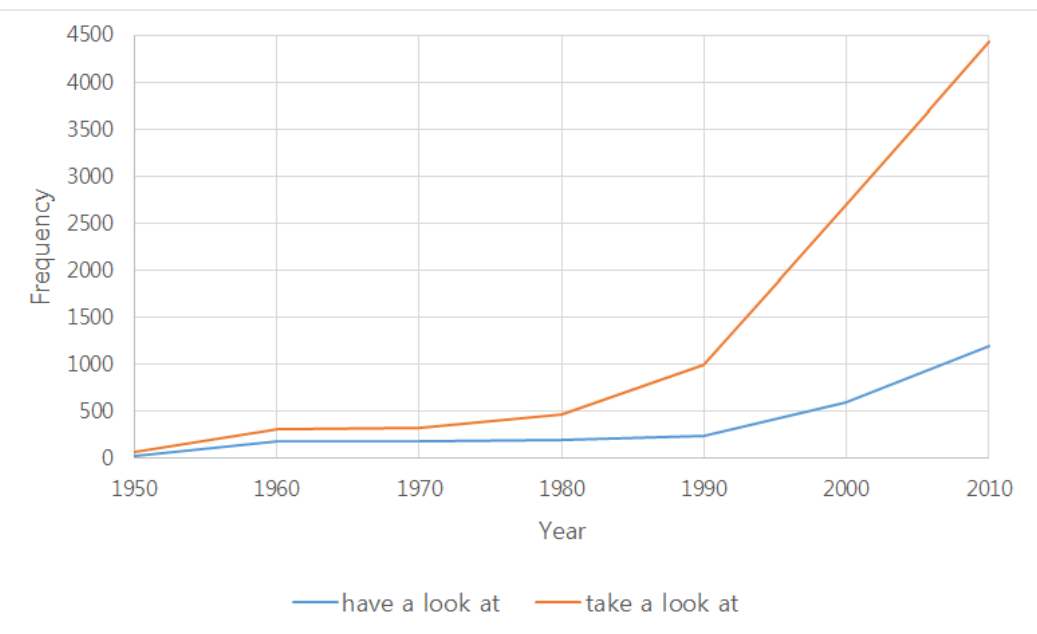

Fig-1: Frequency of have a look at and take a look at in the TV Corpus

It is interesting to note that have a look at shows a steady rise in its frequency for sixty years. More specifically, there was a rise of 1,171 tokens from the 1950 s to the 2010s. More interestingly, have a look at had the lowest frequency in the 1950s (17 tokens), which in turn indicates that it was the least preferred one. On the other hand, have a look at reached a peak $(1,188$ tokens $)$ in the $2010 \mathrm{~s}$, which in turn suggests that it was the most preferred one in all periods. Most importantly, have a look at is the most frequently used in the UK and Ireland. It must be noted, however, that have a look at is the least preferred one in Australia and New Zealand.

It is worth pointing out that there was a steady rise in the figure of take a look at from the 1950s to the 1980s. More specifically, there was a rise of 403 tokens from the 1950s to the 1980s. After this period, there was a dramatic increase in the figure of take a look at. To be more specific, there was an increase of 4,364 tokens from the 1950s to the 2010s. More interestingly, take a look at had the lowest frequency (65 tokens) in the 1950s, which indicates that it was the least preferred one in the 1950s. On the other hand, take a look at reached a peak $(4,429$ tokens) in the 2010 s, which in turn suggests that it was the most preferred one in all periods. As illustrated in Table 1, take a look at was the most frequently used type in America and Canada. On the other hand, it was the least preferred one in Australia and New Zealand. Most importantly, the frequency of take a look at was much higher than that of have a look at from the 1950s to the 2010s. This in turn implies that take a look at was preferred over have a look at all the time. More interestingly, there was a big difference in the figure of two types after the 1990s. To be more specific, there was a sharp increase in the figure of take a look at from the 1990s to the 2010s, whereas there was a gradual increase in the figure of have a look at. In addition to this, it is important to note that have a look at was the most preferred one in the 
UK and Ireland, whereas have a look at was the least preferred one in Australia and New Zealand. It is also important to point out that take a look at was the most preferred one in America and Canada, whereas it was the least preferred one in Australia and New Zealand. We thus conclude that take a look at was favored over have a look at from the 1950 s to the 2015s.

\section{The Movie Corpus}

In this section, we consider the frequency of have a look at and take a look at in the Movie Corpus. Also, we provide a comparative analysis of have a look at and take a look at. Table 2 shows the use and frequency of have a look at and take a look at in the Movie Corpus:

Table-2: Frequency of have a look at and take a look at in the Movie Corpus

\begin{tabular}{|l|l|l|}
\hline Type & Have a look at & Take a look at \\
\hline $1930 \mathrm{~s}$ & 98 & 163 \\
\hline $1940 \mathrm{~s}$ & 154 & 302 \\
\hline $1950 \mathrm{~s}$ & 175 & 324 \\
\hline $1960 \mathrm{~s}$ & 139 & 248 \\
\hline $1970 \mathrm{~s}$ & 122 & 264 \\
\hline $1980 \mathrm{~s}$ & 131 & 406 \\
\hline $1990 \mathrm{~s}$ & 166 & 626 \\
\hline $2000 \mathrm{~s}$ & 346 & 1,234 \\
\hline $2010 \mathrm{~s}$ & 339 & 1,310 \\
\hline All & 1,670 & 4,877 \\
\hline US/CA & 775 & 4,064 \\
\hline UK/IE & 689 & 462 \\
\hline AU/NZ & 103 & 65 \\
\hline Misc & 103 & 286 \\
\hline
\end{tabular}

It is important to note that the frequency of take a look at is much higher than that of have a look at in the Movie Corpus. More specifically, the overall frequency of have a look at is 1,670 tokens, whereas that of take a look at is 4,877 tokens. This in turn shows that take a look at is favored over have a look at in the Movie Corpus. The following graph shows the use and frequency of have a look at and take a look at in the Movie Corpus:

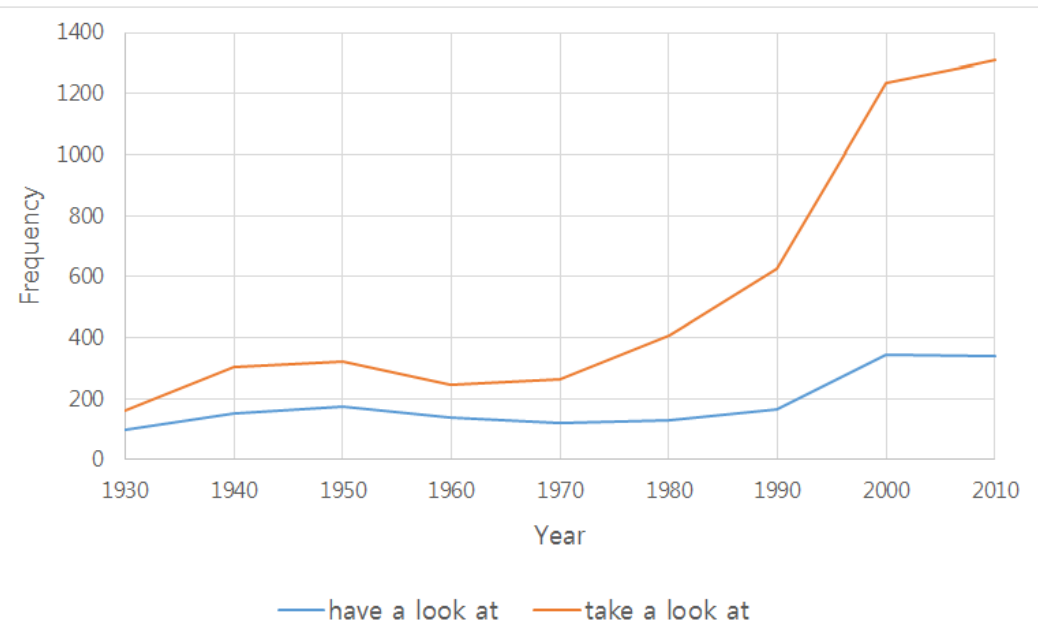

Fig-2: Frequency of have a look at and take a look at in the Movie Corpus

Interestingly, have a look at shows a gradual increase in its figure from the 1930s to the 1950s. More specifically, there was a rise of 77 tokens. More interestingly, there was a sudden decline in the figure of have a look at in the 1960s. There was a decrease of 36 tokens in the 1960s. Again, there was a gradual rise in the figure of have a look at from the 1970s to the 2000s. Interestingly, there was a sudden decrease in the figure of have a look at in the 2010s. That is, there was a decrease of 7 tokens in the 2010s. It is noteworthy that have a look at had the lowest frequency (98 tokens) in the 1930s. This in turn implies that it was the least preferred one in the 1930s. It would be worth mentioning that have a look at reached a peak (346 tokens) in the 2000s. This in turn suggests that it was the most preferred one in the 2000s. It is interesting to note that have a look at was the most preferable one in America and Canada, whereas it was the least preferred one in Australia and New Zealand. 
It is interesting to point out that there was a steady increase in the figure of take a look at from the 1930 s to the 1950s. There was an increase of 161 tokens for twenty years. More importantly, there was a sudden decline of 76 tokens in the 1960s. Again, there was a steady rise in the figure of take a look at from the 1960 s to the 1980s. Most importantly, there was a sharp increase in the figure of take a look at from the 1990s to the 2000s. There was a rise of 608 tokens from the 1990 s to the 2000s. After this period, there was a slight increase in the figure of take a look at in the 2010s. Interestingly, take a look at had the lowest frequency (163 tokens) in the 1930s, which indicates that it was the most undesired type. On the other hand, take a look at reached a peak (1,310 tokens) in the 2010s, which in turn implies that it was the most preferred one in all periods. Interestingly, take a look at was the most frequently used one in America and Canada, whereas it was the least preferred one in Australia and New Zealand. It is worth mentioning that take a look at was preferable to have a look at from the 1930s to the 2010s.

\section{A Collocation Analysis of have a look at and take a look at in the COCA}

In what follows, we consider the collocations of have a look at and take a look at in the COCA. We also provide a comparative analysis of have a look at and take a look at in the COCA. We try to compare the collocation of have a look at and that of take a look at in terms of the software NetMiner. Table 3 shows the collocation of have a look at in the top 25:

Table-3: Collocation of have a look at in the COCA

\begin{tabular}{|l|l|l|}
\hline Number & Collocation of have a look at & Frequency \\
\hline 1 & Have a look at http & 10 \\
\hline 2 & Have a look at things & 3 \\
\hline 3 & Have a look at page & 2 \\
\hline 4 & Have a look at climate & 2 \\
\hline 5 & Have a look at atheism & 1 \\
\hline 6 & Have a look at articles & 1 \\
\hline 7 & Have a look at apple & 1 \\
\hline 8 & Have a look at work & 1 \\
\hline 10 & Have a look at wiki & 1 \\
\hline 11 & Have a look at way & 1 \\
\hline 12 & Have a look at vm-help.com & 1 \\
\hline 13 & Have a look at charity & 1 \\
\hline 14 & Have a look at carbon & 1 \\
\hline 15 & Have a look at breast & 1 \\
\hline 16 & Have a look at biotechnology & 1 \\
\hline 17 & Have a look at senator & 1 \\
\hline 18 & Have a look at Sen & 1 \\
\hline 19 & Have a look at section & 1 \\
\hline 20 & Have a look at Redlands & 1 \\
\hline 21 & Have a look at photography & 1 \\
\hline 22 & Have a look at par & 1 \\
\hline 23 & Have a look at paintings & 1 \\
\hline 24 & Have a look at numbers & 1 \\
\hline 25 & Have a look at Mr & 1 \\
\hline & Have a look at Mom & 1 \\
\hline
\end{tabular}

It is important to note that the expression have a look at http is the most frequently used one in America. This in turn suggests that have a look at http is the most preferred by Americans. As alluded to in Table 3, have a look at http is the most preferable one for Americans, followed by have a look at things, have a look at page (have a look at climate, have a look at atheism), and have a look at articles, in descending order. It is interesting to point out that the everyday expression have a look at page is the third most preferred one in America. On the other hand, have a look at articles is the sixth most preferred one in America. More interestingly, have a look at climate ranks third in the COCA. We thus conclude that have a look at http is the most preferred by Americans.

Now attention is paid to the collocation of take a look at in the COCA. Table 4 shows the frequency of the collocation of take a look at in the top 25: 
Table-4: Collocation of take a look at in the COCA

\begin{tabular}{|c|c|c|}
\hline Number & Collocation of take a look at & Frequency \\
\hline 1 & Take a look at it & 11 \\
\hline 2 & Take a look at http & 10 \\
\hline 3 & Take a look at president & 10 \\
\hline 4 & Take a look at $\mathrm{Mr}$ & 9 \\
\hline 5 & Take a look at history & 8 \\
\hline 6 & Take a look at people & 8 \\
\hline 7 & Take a look at things & 8 \\
\hline 8 & Take a look at chapter & 5 \\
\hline 9 & Take a look at camera & 5 \\
\hline 10 & Take a look at apple & 5 \\
\hline 11 & Take a look at graph & 5 \\
\hline 12 & Take a look at part & 5 \\
\hline 13 & Take a look at issues & 4 \\
\hline 14 & Take a look at politics & 4 \\
\hline 15 & Take a look at ways & 4 \\
\hline 16 & Take a look at Dr & 3 \\
\hline 17 & Take a look at article & 3 \\
\hline 18 & Take a look at films & 3 \\
\hline 19 & Take a look at health & 3 \\
\hline 20 & Take a look at life & 3 \\
\hline 21 & Take a look at page & 3 \\
\hline 22 & Take a look at pictures & 3 \\
\hline 23 & Take a look at weather & 3 \\
\hline 24 & Take a look at websites & 3 \\
\hline 25 & Take a look at adobe & 2 \\
\hline
\end{tabular}

It is interesting to note that the expression take a look at it is the most commonly used one in America. This in turn implies that take a look at it is the most preferable one for Americans. As illustrated in Table 4, take a look at it is the most preferred one in America, followed by take a look at http (take a look at president), take a look at $\mathrm{Mr}$, and take a look at history (take a look at people), in that order. It is worth noting that have a look at http is the most preferred one in America, whereas take a look at http is the second most preferred one. It is also worth pointing out that have a look at $\mathrm{Mr}$ is the sixth most preferred one in America, whereas take a look at $\mathrm{Mr}$ is the fourth most preferred one. More interestingly, have a look at things is the second most preferred one in America, whereas take a look at things is the fifth most preferred one. We thus conclude that the expression take a look at it is the most preferred one in America.

Now let us turn our attention to the visualization of the collocations of have a look at and take a look at in the COCA:

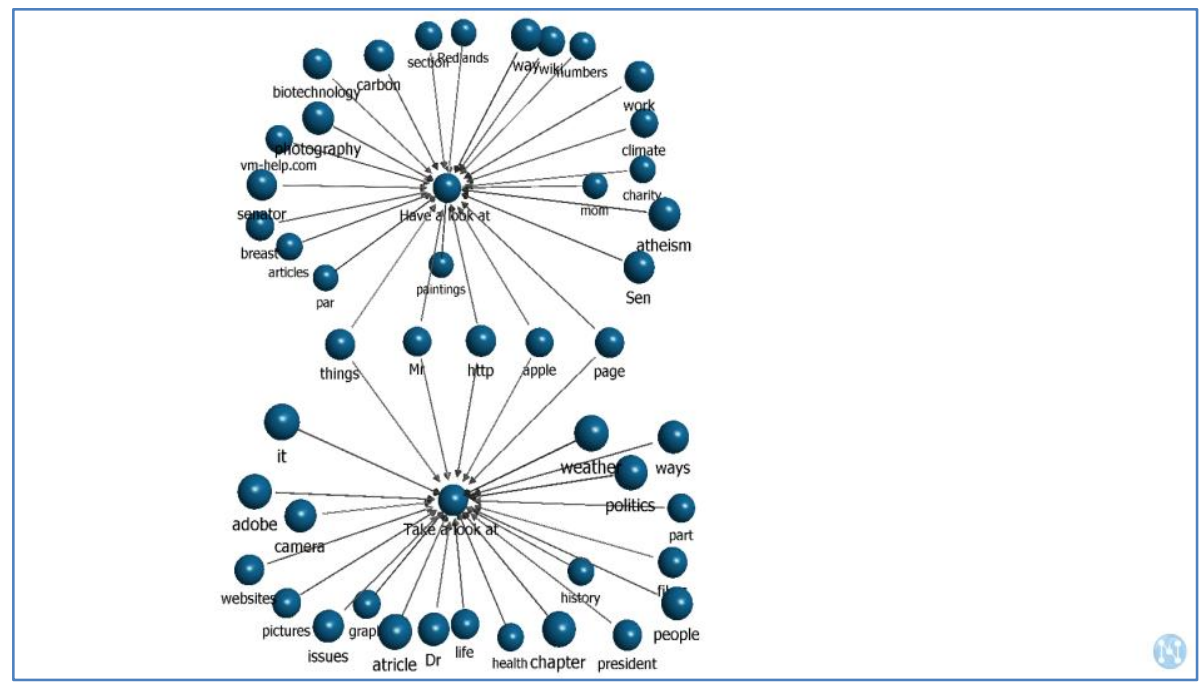

Fig-3: Visualization of the collocations of has a look at and takes a look at in the COCA 
As exemplified in Figure 3, particular nouns are linked to have a look at and take a look at, respectively. Most importantly, five of forty five nouns are linked to both have a look at and take a look at. Five nouns linked to two types are http, Mr, things, apple, and page. This in turn implies that $8.88 \%$ of forty five nouns are the collocations of both have a look at and take a look at. From this it is clear that have a look at and take a look at are low similarity synonyms.

\section{A Collocation Analysis of have a look at and take a look at in the $\mathrm{BNC}$}

In what follows, we examine the collocations of have a look at and take a look at in the BNC. We compare the collocation of have a look at and that of take a look at in the BNC. We also capture the subtle difference between have a look at and take a look at in terms of the software NetMiner. Table 5 shows the frequency of the collocation of have a look at in the top 25:

Table-5: Collocation of have a look at in the BNC

\begin{tabular}{|l|l|l|}
\hline Number & Collocation of have a look at & Frequency \\
\hline 1 & Have a look at number & 3 \\
\hline 2 & Have a look at Mr & 2 \\
\hline 3 & Have a look at things & 2 \\
\hline 4 & Have a look at pages & 2 \\
\hline 5 & Have a look at women & 1 \\
\hline 6 & Have a look at while & 1 \\
\hline 7 & Have a look at wall-papers & 1 \\
\hline 8 & Have a look at mummy & 1 \\
\hline 9 & Have a look at tetanus & 1 \\
\hline 10 & Have a look at swings & 1 \\
\hline 11 & Have a look at SA & 1 \\
\hline 12 & Have a look at rule & 1 \\
\hline 13 & Have a look at Romans & 1 \\
\hline 14 & Have a look at punctuation & 1 \\
\hline 15 & Have a look at percentages & 1 \\
\hline 16 & Have a look at people & 1 \\
\hline 17 & Have a look at mother & 1 \\
\hline 18 & Have a look at jacket & 1 \\
\hline 19 & Have a look at houses & 1 \\
\hline 20 & Have a look at heaven & 1 \\
\hline 21 & Have a look at graphs & 1 \\
\hline 22 & Have a look at fractions & 1 \\
\hline 23 & Have a look at equations & 1 \\
\hline 24 & Have a look at dos & 1 \\
\hline 25 & Have a look at dolly & 1 \\
\hline & & \\
\hline
\end{tabular}

It is significant to note that the expression have a look at number is the most preferred one in the UK. This in turn implies that have a look at number is the most preferable one among the British. As alluded to in Table 5, the expression have a look at number is the most preferred by the British, followed by have a look at Mr (have a look at things, have a look at page), and have a look at women, in descending order. It is important to note that the expressions have a look at things and have a look at page are the second most preferred ones in the UK. Interestingly, have a look at rule and have a look at percentages rank fifth in the $\mathrm{BNC}$. We thus conclude that have a look at number is the most preferable one for the British.

Now let us turn to the collocation of take $a$ look at in the BNC. Table 6 shows the frequency of the collocation of take a look at in the BNC:

Table-6: Collocation of take a look at in the BNC

\begin{tabular}{|l|l|l|}
\hline Number & Collocation of take a look at & Frequency \\
\hline 1 & Take a look at trends & 2 \\
\hline 2 & Take a look at talents & 1 \\
\hline 3 & Take a look at pages & 1 \\
\hline
\end{tabular}

It is interesting to point out that take a look at trends is the most frequently used one in the UK. It implies that the expression take a look at trends is the most preferred one in the UK. As illustrated in Table 6, take a look at trends is the most preferred by the British and followed by take a look at talents (take a look at pages). It would be worth mentioning that have a look at is preferred over take a look at by the British. This 
comes from the fact that the BNC includes only rankthree in the case of the collocation of take a look at. Note, however, that the BNC includes many collocations in the case of have a look at.
Now let us turn our attention to the visualization of the collocations of have a look at and take a look at:

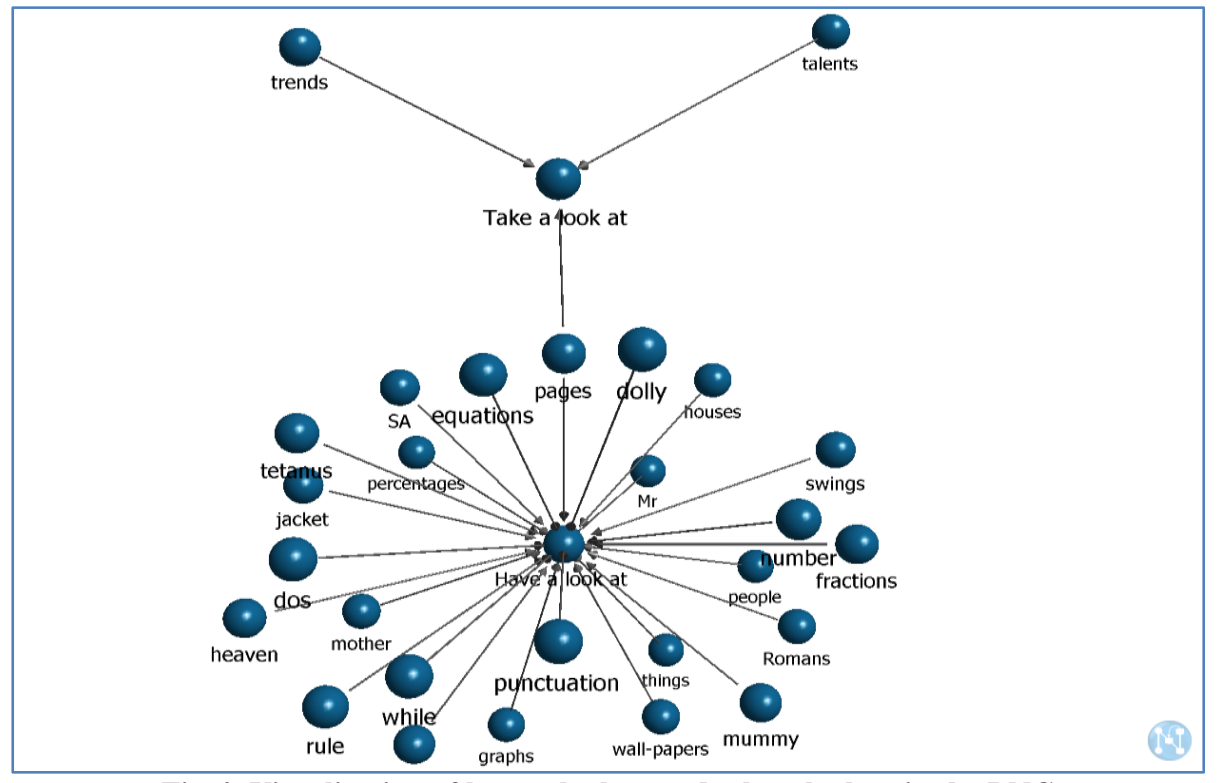

Fig-4: Visualization of have a look at and take a look at in the BNC

As exemplified in Figure 4, twenty seven nouns are linked to have a look at and take a look at, respectively. Most importantly, one of twenty seven nouns is linked to both have a look at and take a look $a t$. This in turn indicates that only $3.7 \%$ of twenty seven nouns are the collocation of have a look at and take a look at. From all of this, it is clear that have a look at and take a look at are low similarity synonyms.

\section{CONCLUSION}

To sum up, we have provided a comparative analysis of have a look at and take a look at in the TV corpus, the Movie Corpus, the COCA, and the BNC. In section 3, we have argued that take a look at is favored over have a look at in the TV Corpus from the 1950s to the 2010s. Interestingly, have a look at reached a peak ( 1,188 tokens) in the 2010s. On the other hand, take a look at reached a peak (4,429 tokens) in the 2010s. This in turn implies that two types are the most preferred ones in the 2010s. In section 4, we have maintained that take a look at is favored over have a look at in the Movie Corpus from the 1930s to the 2010s. More importantly, have a look at reached a peak (346 tokens) in the 2000s, whereas take a look at reached a peak $(1,310$ tokens) in the $2010 \mathrm{~s}$. This in turn shows that two types were the most preferred ones in those periods. In section 5, we have contended that have a look at http is the most preferable one for Americans, whereas take a look at it is the most preferable one for them. Additionally, it is interesting to note that $8.88 \%$ of forty five nouns are the collocations of both have a look at and take a look at in the COCA. In section 6, we have argued that have a look at number is the most preferable one among the British, whereas take a look at trends is the most preferable one among them. Finally, we have shown that only $3.7 \%$ of twenty seven nouns are the collocation of have a look at and take a look at in the BNC.

\section{REFERENCES}

- Murphy, R. (2016). Grammar in Use. Cambridge University Press.

- Murphy, R. (2019). English Grammar in Use. Cambridge University Press.

- British National Corpus (BNC). 1, January 2022. Online https://corpus.byu.edu/bnc

- Corpus of Contemporary American English (COCA). 1, January 2022. Online https://corpus.byu.edu/coca

- Movie Corpus (MC). 1, January 2022. Online https://english-corpora.org /tv/

- TV Corpus (TVC). 1, January 2022. Online https://english-corpora.org /movies/

Cite This Article: Namkil Kang (2022). A Comparative Analysis of have a look at and take a look at in Four Corpora. EAS J Humanit Cult Stud, 4(1), 22-28. 